\title{
The Metaphysics of Consciousness
}

Peter G. Jones

Consciousness is deemed a 'hard' problem in modern scientific consciousness studies wherever it is identified as a metaphysical problem, a problem of first principles, since it is widely assumed that all such problems are intractable to the human intellect. If the problem is sometimes seen as 'easy' this is because not everyone sees it as a metaphysical problem. It was christened 'hard' by David Chalmers on this basis, that Mind-Matter is a metaphysical dilemma and not a problem that can be addressed within the natural sciences. Metaphysics has always known that neither Mind nor Matter is a plausible basis for a fundamental theory, for the failure of extreme metaphysical positions is what makes the subject so difficult in the first place, but philosophers of mind do not always take much notice of metaphysics, and scientists may sometimes be contemptuous. There is a view that metaphysical analysis need not be a constraint on theories of consciousness and this allows many logically indefensible ideas to survive and flourish. But if we are to honestly face up to the 'hard' problem then we must pay metaphysics its rightful dues and concede, with Chalmers, that it cannot be solved within the worldview or mindset that currently prevails in scientific consciousness studies. This is a worldview and mindset for which it has never been possible to solve metaphysical problems and it would make no sense if Mind-Matter were to suddenly become the single exception. In two short articles for The Journal of Consciousness Studies, 'Facing up to the problem of consciousness' and 'Moving on from the problem of consciousness', ${ }^{1}$ Chalmers proposes that we cannot hope to do in scientific consciousness studies what we cannot do in metaphysics and must settle for a nonreductive theory. He names this approach naturalistic dualism. The name suggests that we cannot transcend dualism for a fundamental solution without abanding the conception of Nature that currently prevails in scientific consciousness studies.

Opponents of this pessimistic view, if they are to argue from within the same worldview, must disregard the results of metaphysical analysis and assume that Mind or Matter are, after all, fundamental categories, and hope that we we will eventually discover some scientific evidence that this is the case. This position is not easy to sustain. No amount of scientific evidence would be enough to alter the fact that this profound assumption is logically indefensible and

\footnotetext{
${ }^{1}$ Journal of Consciousness Studies 2(3):200-19 \& 4(1):3-46 (1995)
} 
thus perverse. There can be no evidence to support it unless the universe is paradoxical. If the assumption that Mind or Matter are fundamental categories were logically defensible then metaphysics would be a simple affair. As it is, and it is quite easy to verify, all metaphysical problems are found to be intractable once this assumption has been made. These problems are intricately and intimately connected, and every one of them is found to be 'hard' in exactly the same way. The idea that we might overturn the result of some thousands of years of rational analysis by discovering new scientific evidence is incredible, and a clear misunderstanding of the relationship between physics and metaphysics. From the point of view of the metaphysician to even bother looking for such evidence would be a waste of time. It would simply be a fact that all selective conclusions about the world as a whole are undecidable. This is inevitable, since all selective, extreme or partial metaphysical views are found to be logically indefensible. We cannot choose between them because they are both as bad as each other. Many philosophers have concluded that metaphysics is a waste of time for precisely this reason, that it does not produce a positive result, but this criticism only serves to show that that its result is completely secure. Truly facing up to the problem of consciousness would mean accepting this result of metaphysics as an incontrovertible and directly relevant fact. Naturalistic dualism concedes this fact and is an immediate response to it. We would work only on the easy part of the problem of consciousness and not bother with metaphysics. In this way we can acknowledge the result of metaphysics without having to change our ideas about Nature in any way. This cannot be called facing up to the problem, however, for it is designed as a means of entirely avoiding it. Facing up to the problem would mean conceding that the intractability of Mind-Matter and all other metaphysical problems from within the prevailing worldview is not a plausible coincidence. If it is not a plausible coincidence then it must be the case that it would be impossible to solve the problem of consciousness without solving all other metaphysical problems at the same time. Consciousness would be no more or less important or 'hard' than any other metaphysical question.

The prevailing worldview in scientific consciousness studies has to be the source of the 'hard' problem and its associated metaphysical problems; solipsism, freewill, scepticism, knowledge, origins, ethics and so on. Its failure as a solution for metaphysics is there for all to see. It is logically flawed such that according to reason it must be wrong. It describes a universe that is incomprehensible to us. If it is 'scientific' or 'naturalistic' then it would only be in the same sense as is the theory of philostogen. It renders consciousness 'hard' in exactly the way that Chalmers proposes and demonstrates. Not necessarily intractable, but having no solution that 
can be reconciled with the worldview that currently prevails in the natural sciences and mainstream western metaphysics.

So what is wrong with this worldview? It is often the outcome of an initial assumption stating that metaphysical problems such as Mind-Matter are either decidable or intractable, so that when we fail to decide them we conclude they are intractable. A great many 'hard' problems are created by this assumption. It entails that in respect of the Mind-Matter problem we have only two options. We can assume that it is decidable and endorse one of two counterposed extreme metaphysical positions where all such positions are known to fail, or we can assume that it is intractable and walk away. This is the stark choice with which Chalmers presents us. No other option would be possible for this worldview, for this is the assumption on which it rests.

The assumption that metaphysical questions must be decidable or intractable is not actually necessary for science or naturalism. It is so often made, however, that it is also widely assumed, as a corollary, that the metaphysically flawed and inevitably nonreductive worldview that arises from it must be the only one that can be considered 'scientific' or 'naturalistic'. We must then conclude that in order for a theory to be considered 'scientific' or 'naturalistic' it must fail in metaphysics. And so we are led to the idea that for a naturalistic and scientific worldview we must disregard the results of human reasoning. But, of course, a little thought shows that it is simply not the case that this assumption about metaphysical questions or the worldview that emerges from it is uniquely or even necessarily scientific or naturalistic. If a worldview can legitimately call itself scientific or naturalistic then it would not inevitably follow that it is consistent with the scientific data or survives logical analysis. It may yet be a poorly constructed or hopelessly wrong theory. If it is found to have ramifications inconsistent with logic and reason then calling it 'scientific' or 'naturalistic' is not going to make it any more plausible. Once we have assumed that metaphysical problems are either intractable or decidable, however, then for a 'scientific' or 'naturalistic' theory of consciousness we must stay well clear of metaphysical issues, and we could certainly never revisit this metaphysical assumption to examine it more closely. According to our assumption there would be no point in doing so. We abandoned metaphysics when we start making assumptions instead of doing the calculations. Having made this assumption we have no choice but to conclude, not a little ironically, that the problem of consciousness is too 'hard' for the natural sciences and scientific consciousness studies and that its solution, if there is one, would have to be found elsewhere. Thus Chalmers concludes that if professional consciousness studies is to remain 'scientific' and 
'naturalistic' then the problem of consciousness must be deemed intractable. We would stop looking for a fundamental theory and restrict ourselves to those we would judge scientific or naturalistic, where all theories that would qualify under the current rules are known to fail in metaphysics. In this way we end up with a consciousness studies that we can proudly call scientific and naturalistic and which is doomed to failure for this very reason.

This muddle can be disentangled only if we concede that science is a method and not a set of preconceptions about Nature, and that 'naturalism' is a rejection of the miraculous and paradoxical and not otherwise a limit on our imagination as we try to understand ourselves and our world. We do not have to start by assuming that metaphysical problems are intractable, nor that their solution would have to contradict our reason or be 'unscientific' or 'unnaturalistic'. We would only need to concede that at this time there is something wrong with the way we are looking at these problems and thus at Nature herself. This is clearly the case, for otherwise we would be able to solve these problems and comprehend Nature.

It remains possible, therefore, as a consequence of all this, to argue that the 'easy' part of the problem of consciousness would be the more difficult of the two to solve. It would be an endless task. We would want to know everything we can know about consciousness by the methods of the natural sciences, and then understand the ramifications of this knowledge for cosmology, philosophy, physics, neurophysiology, biology, psychology, psychiatry, artificial intelligence, mathematics, soteriology, religion, the occult, morality, advertising, education, management training, sporting performance and who knows what else. It would be impossible to say what would be required to solve this part of the problem other than a lot of time and money. There is also a deeper issue. If we cannot solve the 'hard' part of the problem in metaphysics then there must be a strict limit on our ability to solve the 'easy' part of it in any other discipline. By such methods we could certainly never hope to learn anything about consciousness that would enable us to decide a metaphysical question, and this places a narrow limit on what we can learn about consciousness as an imputed third-person or intra-subjective phenomenon. Chalmers proposes that we must respect this limit for a naturalistic consciousness studies, one that does not immediately drag us off into the murky waters of metaphysics and mysticism. It would be an impassable barrier to knowledge standing between us and a fundamental theory. But this pessimistic view is not a result of analysis. It is an artefact of an unnecessary assumption about metaphysics and Nature. It is a man-made barrier, not a reason to suppose that a theory which is fundamental, scientific and naturalistic would be impossible. 
The 'hard' part of the problem is more clear-cut, or it can be made so. It is a finite problem, a problem of first principles, a single problem requiring a unique once-and-for-all solution. Crucially, it has not yet been demonstrated that it is intractable. We can concede that it cannot be solved in scientific consciousness studies as currently defined and practised, even insist upon it, and also concede that it is a metaphysical problem, intractable for two millennia or more in the mainstream of European philosophical thought. Yet we can still suppose that it is tractable. This is because it is not yet certain that the prevailing paradigm in European thought and modern scientific consciousness studies, for which these problems are intractable, is in fact naturalistic. Indeed, the intractability of these problems makes it obvious that it is not. If a scientific theory does not describe Nature correctly then it is not naturalistic in anything more than intent. If a theory of consciousness is beset by metaphysical problems then according to reason it is wrong, and if it is wrong then it is not a description of Nature. If naturalism is the claim that all phenomena are natural and subject to natural laws, and if science is defined as a method of data-collection and theory-generation, then unless the universe is paradoxical a truly scientific and naturalistic consciousness studies would encounter no terminally 'hard' problems. Indeed, this would be a crucial test of whether it is naturalistic, that it gives an unproblematic description of Nature. The task for the natural sciences is to establish what is the case, and if we do not yet understand Nature fully then we are forced to remain openminded as to what 'naturalistic' should mean in relation to a fundamental theory of consciousness. If we accept this then the door is thrown open to solutions not available to us when we restrict our ideas about consciousness to exclude those that do not seem 'naturalistic' to us according to some conjectural view of Nature that does not work in metaphysics. For a solution we need not abandon the idea that all phenomena are natural, merely accept that there is much we do not yet know about Nature. Chalmers must be correct in saying that for an orthodox scientific consciousness studies we must settle for a nonreductive theory, for if we set out to pursue the problem of consciousness to its end we cannot avoid becoming enmired in metaphysics and religion. But this would mean that the solution for consciousness would have to be sought beyond consciousness studies, in metaphysics and religion. This does not seem like progress. Do we really want to emasculate consciousness studies in this way? It would then be beyond the remit of scientific consciousness studies to declare consciousness an 'easy' or a 'hard' problem, since which it is would depend entirely on how difficult it would be to solve in metaphysics or religion. Narrowing the scope of consciousness studies in this way in order to protect an incomprehensible naturalism would not be necessary if we say that science is a 
method and not a theory, and that naturalism is a commitment to the laws of Nature and not a statement of what they are.

There is then some reason to think that the 'hard' problem might not be terminally difficult. The difficulty may be only that our incorrect preconceptions about Nature make it so. Is this not certain? It is not as if we need more data. If we cannot solve this problem then where else can the fault lie than in our preconceptions about Nature? For the natural sciences we usually begin by assuming that 'Mind' and 'Matter' are categories within which we can place all natural phenomena. This is to assume that we know a lot more about Nature than we actually do. It is a metaphysical assumption, a gamble, and there is nothing particularly scientific or naturalistic about it. Only if we allow ourselves to be dragged into metaphysics and religion can we abandon this assumption, for here we find a different idea. We need not abandon naturalism and science, only certain orthodoxies, preconceptions, assumptions and dogmas. As for whether we would be abandoning our traditional metaphysics, the intractability of the problem of consciousness and other related problems is conclusive proof that our usual metaphysical approach is fundamentally flawed. Why would we not want to abandon it? Every one of its problems is caused by the assumption that some distinction or categorisation such as Mind-Matter is fundamental, while the only available solution for them would be to assume otherwise. The problem is only this. In order to assume that Mind-Matter and all such distinctions are emergent we would have to abandon the assumption that metaphysical problems are decidable or intractable, and this cannot be done within the prevailing paradigm.

We can briefly examine one case to illustrate the possibility of a different approach. In one of its guises the problem of consiousness appears as the problem of matter-mind causation. The idea that a corporeal phenomenon can act causally on a mental phenomenon goes largely unquestioned in the sciences, but it is no less problematic than the idea that mind can act on matter. The mismatch of phenomena is the same in both directions. One is material and the other is not, and we have no theory for how this gap can be bridged by any form of causation even at the moment of their creation. Not even the existence of consciousness can be established in physics. But we can use our reason. In metaphysics we find it impossible to reduce Mind and Matter one to the other or to make them identical, and their causal relationship seems to be miraculous. Unless, that is, we hypothesise another phenomenon, something that cannot be classified as Mind or Matter. Given an extra ingredient we no longer have to suppose that there is a paradoxical causal link between the physical and the mental worlds, nor that one is fundamental and the other emergent. This is a plausible solution because 
it represents a paradigmatic change of approach. For the prevailing view we must assume that one or both of Mind and/or Matter takes precedence in the evolution of the universe. Usually one would be original and cause the other. As this idea does not make sense all sorts of problems arise for it that seem impregnable to human reason and cannot be solved in metaphysics, consciousness studies or physics. It cannot be a correct assumption. The present state of western academic metaphysics and consciousness studies represents a highly successful reductio argument against it. With a third phenomenon ontologically prior to mental and corporeal phenomena, however, we can suppose that these two emerge as dual-aspects in mutual-dependence and can be described within a dual-aspect theory very much as Chalmers proposes. We need not imagine that either can exist independently, that one or the other sprang into existence from nothing at all, nor that one directly causes the other. They would share a common source and a mediated relationship.

This is mysticism, of course, the 'nondual' or advaita view, the doctrine of 'dependent origination ', the realm of Lao Tsu's Tao. In the context of consciousness studies it has been called 'relative phenomenalism'. It would also be very nearly the solution proposed by Chalmers' with his dual-aspect theory of information, where these two aspects would be the mental and the corporeal. It is only that now it would contain an extra ingredient, a third theoretical primitive, one that would allow the theory to be fundamental. As well as the parts there would be the whole. So similar is this to Chalmers' theory, being merely an extension of it, and so obvious is it that it might be a solution for the problem, that it can only be an unnecessarily narrow interpretation of the words 'scientific' and 'naturalistic' that prevent it from being a well-regarded theory in scientific consciousness studies. In one of its guises it is Middle Way Buddhism, and so the reason cannot be a lack of publicity. Its lack of credibility seems more likely to be due to certain assumptions about science and naturalism that prevent those who make them from seeing the full possibilities of Nature.

It is at least possible, therefore, that the 'hard' problem of consciousness is a tractable one and even, at the level of principles, a fairly straightforward one. The solution would be to assume that the Buddha and Lao Tsu knew what they were talking about. It is often forgotten that they predicted the Mind-Matter problem, our inability to reduce this dualism, and all such metaphysical dilemmas, when they told us that the categories of thought are not fundamental, that all distinctions are emergent. Even if we do not believe them we can at least be sure, after all this time, that Chalmers is right, and that it would be impossible to gainsay them. 